\title{
On the striction curves of Mannheim Frenet ruled surfaces in $E^{3}$
}

\author{
Seyda Kilicoglu ${ }^{1}$, Suleyman Senyurt ${ }^{2}$ and Huseyin Kocayigit ${ }^{3}$ \\ ${ }^{1}$ Başkent University, Faculty of Education, Ankara, Turkey \\ ${ }^{2}$ Ordu University, Faculty of Arts and Sciences, Ordu, Turkey \\ ${ }^{3}$ Celal Bayar University, Faculty of Art And Science, Manisa, Turkey
}

Received: 2 June 2017, Accepted: 1 December 2018

Published online: 31 December 2018.

\begin{abstract}
In this paper we consider special ruled surfaces associated to Mannheim curve $\alpha$ and Mannheim partner $\alpha^{*}$, with $k_{1} \neq 0$. They are called as Frenet ruled surface (FRS) and Mannheim Frenet ruled surfaces (MFRS), cause of their generators are the renet vector fields of Mannheim curve pair $\left(\alpha, \alpha^{*}\right)$. First we give the tangent vector fields of the striction curves of Mannheim Frenet ruled surfaces in terms of the Frenet apparatus of Mannheim curve $\alpha$. Further we give only one matrix in which we can examine sixteen position of these tangent vector fields, such that we can say the conditions of perpendicularity of these tangent vector fields.
\end{abstract}

Keywords: Mannheim curve pair, striction curves, rules surfaces, Frenet ruled surface.

\section{Introduction}

A curve is called a Mannheim curve if and only if $\frac{k_{1}}{\left(k_{1}^{2}+k_{2}^{2}\right)}$ is a non-zero constant, $k_{1}$ is the curvature and $k_{2}$ is the torsion by A. Mannheim in 1878. Liu and Wang in [10] give a new definition as; if the principal normal vector of first curve and binormal vector of second curve are linearly dependent, then first curve is called Mannheim curve, and the second curve is called Mannheim partner curve. Also they called these new curves as Mannheim pair curve. Let $\alpha: I \rightarrow E^{3}$ be the $C^{2}-$ class differentiable unit speed curve with the quantities $\left\{V_{1}, V_{2}, V_{3}, \tilde{D}, k_{1}, k_{2}\right\}$ are collectively Frenet-Serret apparatus of the curve $\alpha: I \rightarrow E^{3}$. Where $\tilde{D}(s)=\frac{k_{2}}{k_{1}}(s) V_{1}(s)+V_{3}(s)$ under the condition that $k_{1} \neq 0$ is called the modified Darboux vector field of $\alpha$ [5]. Let $\alpha^{*}: I \rightarrow E^{3}$ be second curve and $\left\{V_{1}^{*}\left(s^{*}\right), V_{2}^{*}\left(s^{*}\right), V_{3}^{*}\left(s^{*}\right)\right\}$ be the Frenet frames of the curve $\alpha^{*}$ If the principal normal vector $V_{2}$ of the curve $\alpha$ is linearly dependent on the binormal vector $V_{3}^{*}\left(s^{*}\right)$ of the curve $\alpha^{*}$, then the pair $\left\{\alpha, \alpha^{*}\right\}$ is a Mannheim pair, so $\alpha$ is called a Mannheim curve and $\alpha^{*}$ called Mannheim partner curve of $\alpha$ where $\measuredangle\left(V_{1}, V_{1}^{*}\right)=0 \neq \frac{\pi}{2}$. Mannheim partner curve of $\alpha$ can be represented $\alpha^{*}(s)=\alpha^{*}\left(s^{*}\right)+\lambda\left(s^{*}\right) V_{3}^{*}\left(s^{*}\right)$ for some function $\lambda$, since $V_{2}$ and $V_{3}^{*}$ are linearly dependent, Equation can be rewritten as $\alpha^{*}(s)=\alpha(s)-\lambda V_{2}(s)[11]$, where $\lambda=\frac{-k_{1}}{k_{1}^{2}+k_{2}^{2}}$ is a non-zero constant. Frenet-Serret apparatus of Mannheim partner curve $\alpha^{*}$, based in Frenet-Serret vectors of Mannheim curve $\alpha$ are

$$
V_{1}^{*}=\cos \theta V_{1}-\sin \theta V_{3}, V_{2}^{*}=\sin \theta V_{1}+\cos \theta V_{3}, V_{3}^{*}=V_{2}, \tilde{D}^{*}=\frac{\sqrt{k_{1}^{2}+k_{2}^{2}}}{\theta^{\prime}}\left(\cos \theta V_{1}-\sin \theta V_{3}\right)+V_{2}
$$


The curvature and the torsion have the following equalyties, $k_{1}^{*}=-\frac{d \theta}{d s^{*}}=\frac{\theta^{\prime}}{\cos \theta}$ and $k_{2}^{*}=\frac{k_{1}}{\lambda k_{2}}$, we use dot to denote the derivative with respect to the arc length parameter of the curve $\alpha$. Also $\frac{d s}{d s^{*}}=\frac{1}{\sqrt{1+\lambda k_{2}}}=\frac{1}{\cos \theta}$ [11]. Also we need the following equalities;

$$
k_{1}^{*}=\frac{k_{1} \theta^{\prime}}{\lambda k_{2} \cos \theta}, \eta^{*}=k_{1}^{* 2}+k_{2}^{* 2}=\frac{\theta^{\prime 2} \lambda^{2} k_{2}^{2} \cos ^{2} \theta}{\lambda^{2} k_{2}^{2} \cos ^{2} \theta}, \gamma=\frac{k_{1}^{*}}{k_{1}^{* 2}+k_{2}^{* 2}}=\frac{\lambda^{2} k_{2}^{2} \theta^{\prime} \cos \theta}{\lambda^{2} k_{2}^{2} \theta^{\prime 2}+k_{1}^{2} \cos ^{2} \theta}
$$

\section{Frenet ruled surface and Striction curves}

A ruled surface is one which can be generated by the motion of a straight line in Euclidean 3-space, [1]. Frenet ruled surface is one which can be generated by the motion of a Frenet vector of any curve in Euclidean 3-space: We bring here the famous example of L. K. Graves, so called the B-scroll, in [3]. Involute B-scroll is defined and based on normal vector fields are examined in [6]. The differential geometric elements of the involute $\tilde{D}$ scroll are examined in [13]. The positions of normal vector fields of BFRS and their striction curves are examined in [7], [8]. Also in [12] Mannheim offsets of ruled surfaces are defined and characterized. In this subsection tangent, normal, binormal, Darboux ruled surfaces of any curve are collectively named Frenet ruled surfaces (FRS) [2]. They have the following equations. In the Euclidean 3 -space, let $\alpha(s)$ be the arclengthed curve. The equations

$$
\varphi_{1}\left(s, u_{1}\right)=\alpha(s)+u_{1} V_{1}(s), \varphi_{2}\left(s, u_{2}\right)=\alpha(s)+u_{2} V_{2}(s), \varphi_{3}\left(s, u_{3}\right)=\alpha(s)+u_{3} V_{3}(s), \varphi_{4}\left(s, u_{4}\right)=\alpha(s)+u_{4} \tilde{D}(s),
$$

are the parametrization of Frenet ruled surfaces which are called tangent ruled surface, normal ruled surface, binormal ruled surface, Darboux ruled surface, respectively in [2]. The striction point on a ruled surface $\varphi(s, v)=\alpha(s)+v e(s)$ is the foot of the common normal between two consecutive generators (or ruling). The set of striction points defines the striction curve given by

$$
c(s)=\alpha(s)-\frac{\left\langle\alpha_{s}, e_{s}\right\rangle}{\left\langle e_{s}, e_{s}\right\rangle} e(s)
$$

[1], [4]. The striction curves of four Frenet ruled surfaces is given by the following matrix

$$
\left[\begin{array}{c}
c_{1}-\alpha \\
c_{1}-\alpha \\
c_{1}-\alpha \\
c_{1}-\alpha
\end{array}\right]=\left[\begin{array}{ccc}
0 & 0 & 0 \\
0 & \frac{k_{1}}{k_{1}^{2}+k_{2}^{2}} & 0 \\
0 & 0 & 0 \\
\frac{-k_{2}}{k_{1}\left(\frac{k_{2}}{k_{1}}\right)} & 0 & \frac{-1}{\left(\frac{k_{2}}{k_{1}}\right)}
\end{array}\right]\left[\begin{array}{c}
V_{1} \\
V_{2} \\
V_{3}
\end{array}\right]
$$

[9]. Tangent vector fields $T_{1}, T_{2}, T_{3}$ and $T_{4}$ of striction curves of Frenet ruled surfaces, respectively are given by

$$
\begin{gathered}
{[T]=[A][V]=\left[\begin{array}{l}
T_{1} \\
T_{2} \\
T_{3} \\
T_{4}
\end{array}\right]=\left[\begin{array}{lll}
1 & 0 & 0 \\
a & b & c \\
1 & 0 & 0 \\
d & 0 & e
\end{array}\right]\left[\begin{array}{l}
V_{1} \\
V_{2} \\
V_{3}
\end{array}\right]} \\
a=\frac{k_{2}^{2}}{\eta\left\|c_{2}^{\prime}(s)\right\|}, b=\frac{\left(\frac{k_{1}}{\eta}\right)^{\prime}}{\left\|c_{2}^{\prime}(s)\right\|}, c=\frac{k_{1} k_{2}}{\eta\left\|c_{2}^{\prime}(s)\right\|} d=\frac{\mu-\mu^{\prime}-\frac{k_{2}}{k_{1}}}{\mu\left\|c_{4}^{\prime}(s)\right\|}, e=\frac{\mu^{\prime}}{\mu^{2}\left\|c_{4}^{\prime}(s)\right\|},
\end{gathered}
$$


where $\eta=k_{1}^{2}+k_{2}^{2}$ and $\mu=\left(\frac{k_{2}}{k_{1}}\right)^{\prime}[8]$

\section{Mannheim Frenet ruled surfaces and striction curves}

Definition 1. Let $\left\{\alpha^{*}, \alpha\right\}$ be Mannheim curve pair with $k_{1} \neq 0$ and $k_{2} \neq 0$. The equations of the ruled surfaces

$$
\begin{array}{ll}
\varphi_{1}^{*}\left(s^{*}, v_{1}\right)=\alpha^{*}(s)+v_{1} V_{1}^{*}\left(s^{*}\right), & \varphi_{2}^{*}\left(s^{*}, v_{2}\right)=\alpha^{*}(s)+v_{2} V_{2}^{*}\left(s^{*}\right), \\
\varphi_{3}^{*}\left(s^{*}, v_{3}\right)=\alpha^{*}(s)+v_{3} V_{3}^{*}\left(s^{*}\right), & \varphi_{4}^{*}\left(s^{*}, v_{4}\right)=\alpha^{*}(s)+v_{4} \tilde{D}^{*}\left(s^{*}\right)
\end{array}
$$

are the parametrization of Frenet ruled surface of Mannheim partner $\alpha^{*}$. Further we can give the new definitions of these surfaces as in the following way; are called Mannheim tangent ruled surface, Mannheim normal ruled surface, Mannheim binormal ruled surface and Mannheim Darboux ruled surface respectively. They are collectively Mannheim Frenet ruled surface (MFRS).

Theorem 1. The equatinos of the striction curves of four Mannheim Frenet ruled sur faces along the Mannheim partner $\alpha^{*}$ in terms of Frenet apparatus of Mannheim curve $\alpha$

$$
\left[\begin{array}{l}
c_{1}^{*}-\alpha \\
c_{2}^{*}-\alpha \\
c_{3}^{*}-\alpha \\
c_{4}^{*}-\alpha
\end{array}\right]=\left[\begin{array}{ccc}
0 & -\lambda & 0 \\
\frac{\lambda^{2} k_{2}^{2} \theta^{\prime} \cos \theta \sin \theta}{\lambda^{2} k_{2}^{2} \theta^{\prime 2}+k_{1}^{2} \cos ^{2} \theta} & -\lambda & \frac{\lambda^{2} k_{2}^{2} \theta^{\prime} \cos ^{2} \theta}{\lambda^{2} k_{2}^{2} \theta^{\prime 2}+k_{1}^{2} \cos ^{2} \theta} \\
0 & -\lambda & 0 \\
-\frac{k_{1}}{\lambda k_{2} \theta^{\prime} \beta^{\prime}} \cos \theta & -\left(\lambda+\frac{k_{1}}{\lambda k_{2} \theta^{\prime} \beta^{\prime}}\right) & \frac{k_{1}}{\lambda k_{2} \theta^{\prime} \beta^{\prime}} \cos ^{2} \theta \sin \theta
\end{array}\right]\left[\begin{array}{c}
V_{1} \\
V_{2} \\
V_{3}
\end{array}\right], \quad \beta=\frac{k_{1} \cos \theta}{\lambda k_{2} \theta^{\prime}} .
$$

Proof. It is trivial that four the striction curves of four Frenet ruled surfaces along Mannheim partner curve $\alpha^{*}$ are

$$
\begin{aligned}
& c_{1}^{*}=\alpha(s)-\lambda V_{2}(s), \\
& c_{2}^{*}=\alpha(s)+\frac{k_{1}^{*}}{\left(k_{1}^{* 2}+k_{2}^{* 2}\right)} \sin \theta V_{1}-\lambda V_{2}(s)+\frac{k_{1}^{*}}{\left(k_{1}^{* 2}+k_{2}^{* 2}\right)} \cos \theta V_{3}, \\
& c_{3}^{*}=\alpha(s)-\lambda V_{2}(s), \\
& c_{4}^{*}=\alpha(s)-\frac{\frac{k_{2}^{*}}{k_{1}^{*}}}{\left(\frac{k_{2}^{*}}{k_{1}^{*}}\right)^{\prime}} \cos \theta V_{1}-\left(\lambda+\frac{1}{\left(\frac{k_{2}^{*}}{k_{1}^{*}}\right)^{\prime}}\right) V_{2}+\frac{\frac{k_{2}^{*}}{k_{1}^{*}}}{\left(\frac{k_{2}^{*}}{k_{1}^{*}}\right)^{\prime}} \sin \theta V_{3} .
\end{aligned}
$$

The striction curves of four Frenet ruled surfaces along the Mannheim partner $\alpha^{*}$ are given by the following matrix

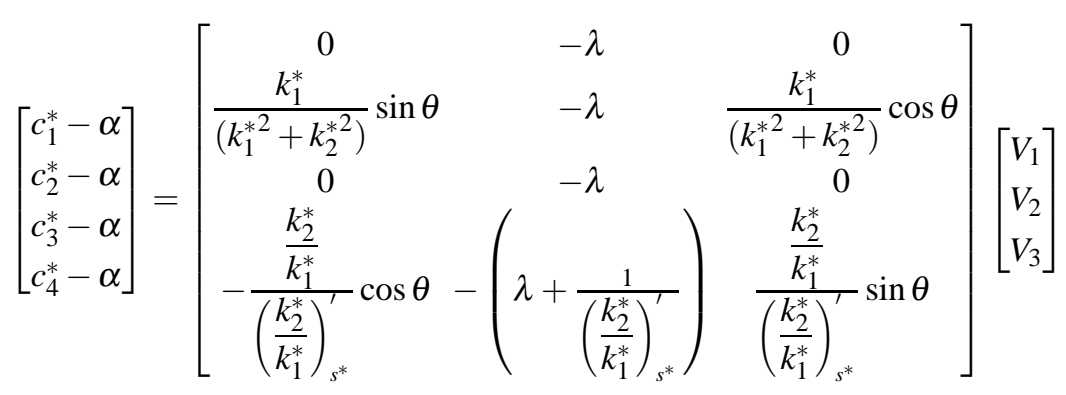

by using $\frac{k_{2}^{*}}{k_{1}^{*}}=\frac{k_{1} \cos \theta}{\lambda k_{2} \theta^{\prime}}=\beta$ and derivation $\left(\frac{k_{2}^{*}}{k_{1}^{*}}\right)_{s^{*}}^{\prime}=\left(\frac{k_{1} \cos \theta}{\lambda k_{2} \theta^{\prime}}\right) \frac{1}{\cos \theta}=\frac{\beta^{\prime}}{\cos \theta}=\mu^{*}$ we have the proof. 
Proposition 1. Tangent vector fields $T_{1}{ }^{*}, T_{2}{ }^{*}, T_{3}{ }^{*}, T_{4}{ }^{*}$ of striction curves along Frenet ruled surface in terms of Frenet apparatus by themself are given by

$$
\begin{gathered}
{\left[T^{*}\right]=\left[\begin{array}{c}
T_{1}{ }^{*} \\
T_{2}{ }^{*} \\
T_{3}{ }^{*} \\
T_{4}{ }^{*}
\end{array}\right]=\left[\begin{array}{ccc}
1 & 0 & 0 \\
a^{*} & b^{*} & c^{*} \\
1 & 0 & 0 \\
d^{*} & 0 & e^{*}
\end{array}\right]\left[\begin{array}{l}
V_{1}{ }^{*} \\
V_{2}{ }^{*} \\
V_{3}{ }^{*}
\end{array}\right] .} \\
a^{*}=\frac{k_{2}{ }^{* 2}}{\eta^{*}\left\|c_{2}{ }^{\prime}(s)\right\|^{*}}, b^{*}=\frac{\left(\frac{k_{2}{ }^{*}}{\left.k_{1}\right)^{\prime}}\right.}{\left\|c_{2}{ }^{\prime}(s)\right\|^{*}}, c^{*}=\frac{k_{1}{ }^{*} k_{2}{ }^{*}}{\eta^{*}\left\|c_{2}{ }^{\prime}(s)\right\|^{*}}, d^{*}=\frac{\mu^{* 2}-\mu^{*} \mu^{* \prime} \frac{k_{2}{ }^{*}}{k_{1}{ }^{*}} \mu^{*}}{\mu^{*}\left\|c_{4}{ }^{\prime}(s)\right\|^{*}}, \\
e^{*}=\frac{\mu^{* \prime}}{\mu^{*}\left\|c_{4}{ }^{\prime}(s)\right\|^{*}}, \eta^{*}=k_{1}{ }^{*}+k_{2}{ }^{*}, \quad \mu^{*}=\left(\frac{k_{2}{ }^{*}}{k_{1}{ }^{*}}\right)^{\prime}
\end{gathered}
$$

and

$$
[T]=[A][V]\left[\begin{array}{lll}
1 & 0 & 0 \\
a & b & c \\
1 & 0 & 0 \\
d & 0 & e
\end{array}\right]\left[\begin{array}{l}
V_{1} \\
V_{2} \\
V_{3}
\end{array}\right]
$$

Theorem 2. The product of tangent vector fields $T_{1}, T_{2}, T_{3}, T_{4}$ and tangent vector fields $T_{1}{ }^{*}, T_{2}{ }^{*}, T_{3}{ }^{*}, T_{4}{ }^{*}$ of striction curves of FRS and MFRS are given in terms of Frenet apparatus by

$$
[T]\left[T^{*}\right]^{T}=\left[\begin{array}{cccc}
\cos \theta & a^{*} \cos \theta+b^{*} \sin \theta & \cos \theta & d * \cos \theta \\
a \cos \theta-c \sin \theta & \sin \theta\left(b^{*} a-a^{*} c\right)+\cos \theta\left(a^{*} a+b^{*} c\right)+c^{*} b & a \cos \theta-c \sin \theta & d^{*}(a \cos \theta-c \sin \theta)+b e^{*} \\
\cos \theta & a^{*} \cos \theta+b^{*} \sin \theta & \cos \theta & d^{*} \cos \theta \\
d \cos \theta-e \sin \theta & \sin \theta\left(b^{*} d-a^{*} e\right)+\cos \theta\left(a^{*} d+b^{*} e\right) & d \cos \theta-e \sin \theta & d^{*}(d \cos \theta-e \sin \theta)
\end{array}\right]
$$

Proof. Let $[T]=[A][V]$ and $\left[T^{*}\right]=\left[A^{*}\right]\left[V^{*}\right]$ hence

$$
\begin{aligned}
{[T]\left[T^{*}\right]^{T}=} & {[A][V]\left(\left[A^{*}\right]\left[V^{*}\right]\right)^{T}=[A]\left([V]\left[V^{*}\right]^{T}\right)\left[A^{*}\right]^{T} } \\
= & {\left[\begin{array}{lll}
1 & 0 & 0 \\
a & b & c \\
1 & 0 & 0 \\
d & 0 & e
\end{array}\right]\left[\begin{array}{l}
V_{1} \\
V_{2} \\
V_{3}
\end{array}\right]\left(\left[\begin{array}{ccc}
1 & 0 & 0 \\
a^{*} & b^{*} & c^{*} \\
1 & 0 & 0 \\
d^{*} & 0 & e^{*}
\end{array}\right]\left[\begin{array}{c}
V_{1}^{*} \\
V_{2}^{*} \\
V_{3}^{*}
\end{array}\right]\right)^{T}=\left[\begin{array}{crr}
\cos \theta & \sin \theta & 0 \\
a \cos \theta-c \sin \theta & a \sin \theta+c \cos \theta & b \\
\cos \theta & \sin \theta & 0 \\
d \cos \theta-e \sin \theta d \sin \theta+e \cos \theta & 0
\end{array}\right]\left[\begin{array}{llll}
1 & a^{*} & 1 & d^{*} \\
0 & b^{*} & 0 & 0 \\
0 & c^{*} & 0 & e^{*}
\end{array}\right] }
\end{aligned}
$$

hence we have the proof. The matrix product of Frenet vector fields of the Mannheim partner curve $a^{*}$; Mannheim curve a has the following matrix form;

$$
\left[\begin{array}{l}
V_{1} \\
V_{2} \\
V_{3}
\end{array}\right]\left[\begin{array}{l}
V_{1}^{*} \\
V_{2}^{*} \\
V_{3}^{*}
\end{array}\right]^{T}=\left[\begin{array}{ccc}
\cos \theta & \sin \theta & 0 \\
0 & 0 & 1 \\
-\sin \theta & \cos \theta & 0
\end{array}\right]
$$

Proposition 2. The four pairs of Frenet ruled surface and Mannheim Frenet ruled surface have not striction curves with perpendicular tangent vector fields, these are; Tangent ruled surface and Mannheim tangent ruled surface, Tangent ruled surface and Mannheim binormal ruled surface, Binormal ruled surface and Mannheim tangent ruled surface, Binormal ruled surface and Mannheim binormal ruled surface. 
Proposition 3. Normal ruled surface and Mannheim tangent ruled surface; Normal ruled surface and Mannheim binormal ruled surface have striction curves with perpendicular tangent vector fields if

$$
\tan \theta=\frac{k_{1}}{k_{2}}
$$

Proposition 4. Darboux ruled surface and Mannheim tangent ruled surface and Darboux ruled surface and Mannheim binormal ruled surface have striction curves with perpendicular tangent vector fields if

$$
\tan \theta=\frac{\left(\mu-\mu^{\prime}-\frac{k_{1}}{k_{2}}\right) \mu}{\mu^{\prime}} \quad \text { where }\left(\frac{k_{1}}{k_{2}}\right)^{\prime}
$$

Proposition 5. Tangent ruled surface and Mannheim normal ruled surface; binormal ruled surface and Mannheim normal ruled surface of have striction curves with perpendicular tangent vector fields if

$$
\tan \theta=\frac{-k_{1}^{2} \cos ^{3} \theta}{\left(\lambda^{2} k_{2}^{2} \theta^{\prime 2}+k_{1}^{2} \cos ^{2} \theta\right)\left(\frac{\lambda^{2} k_{2}^{2} \theta^{\prime 2} \cos \theta}{\lambda^{2} k_{2}^{2} \theta^{\prime 2}+k_{1}^{2} \cos ^{2} \theta}\right)^{\prime}}
$$

Theorem 3. Tangent ruled surface and Mannheim Darboux ruled surface; Binormal ruled surface and Mannheim Darboux ruled surface have striction curves with perpendicular tangent vector fields if

$$
\frac{\beta^{\prime}}{\cos \theta}-\left(\frac{\beta^{\prime}}{\cos \theta}\right)^{\prime} \frac{1}{\cos \theta}-\beta=0 ; \quad \cos \theta \neq 0, \quad \text { where } \quad \beta=\frac{k_{1} \cos \theta}{\lambda k_{2} \cos \theta^{\prime}}=\frac{k_{2}{ }^{*}}{k_{1}{ }^{*}}
$$

Proof. Since $\left\langle T_{1},\left(T_{4}\right)^{*}\right\rangle=\left\langle T_{3},\left(T_{4}\right)^{*}\right\rangle=d^{*} \cos \theta$, and under the orthogonality condition $d^{*} \cos \theta=0$, hence

$$
\frac{\mu^{*}-\mu^{* \prime}-\frac{k_{2}{ }^{*}}{k_{1}{ }^{*}}}{\mu^{*}\left\|c_{4}{ }^{\prime \prime}(s)\right\|}=0 \Rightarrow m u^{*}-\mu^{* \prime}{ }_{s^{*}}-\frac{k_{2}{ }^{*}}{k_{1}{ }^{*}}=0 \Rightarrow \frac{\beta^{\prime}}{\cos \theta}-\left(\frac{\beta^{\prime}}{\cos \theta}\right)^{\prime} \frac{1}{\cos \theta}-\beta=0 \text { where } \mu^{* \prime}{ }_{s^{*}}=\left(\frac{\beta^{\prime}}{\cos \theta}\right)^{\prime} \frac{1}{\cos \theta}
$$

Theorem 4. Normal ruled surface and Mannheim normal ruled surface have striction curves with perpendicular tangent vector fields under the condition

$$
\frac{-k_{1}}{\lambda} \cos \theta+\left(\frac{\lambda k_{2}^{2} \gamma \gamma^{\prime}}{-k_{1} \cos \theta}+\frac{k_{2}^{2} k_{2}}{\lambda k_{2}^{2}}\right) \sin \theta+\frac{\left(\frac{k_{2}}{k_{1}}\right)^{\prime} k_{1} \cos \theta^{\prime}}{\lambda k_{2} \cos \theta}-\lambda k_{2}^{2} \gamma \gamma^{\prime}=0
$$

Proof. Since $\left\langle T_{2},\left(T_{2}\right)^{*}\right\rangle=a^{*}(a \cos \theta-c \sin \theta)+b^{*}(a \cos \theta+c \sin \theta)+b c^{*}$, and under the orthogonality condition $a^{*}(a \cos \theta-c \sin \theta)+b^{*}(a \cos \theta+c \sin \theta)+b c^{*}=0$, hence

$$
\begin{array}{r}
\frac{k_{2}{ }^{* 2}}{\eta^{*}\left\|c_{2}{ }^{\prime}(s)\right\|^{*}}(a \cos \theta-c \sin \theta)+\frac{\left(\frac{k_{1}{ }^{*}}{\eta^{*}}\right)^{\prime}}{\eta^{*}\left\|c_{2}{ }^{\prime}(s)\right\|^{*}} a \cos \theta+c \sin \theta+b \frac{k_{1}{ }^{*} k_{2}{ }^{*}}{\eta^{*}\left\|c_{2}{ }^{\prime}(s)\right\|^{*}}=0, \\
\frac{-k_{1}}{\lambda} \cos \theta+\left(\frac{\lambda k_{2}{ }^{2} \gamma \gamma^{\prime}}{-k_{1} \cos \theta}+\frac{k_{2}{ }^{2} k_{2}}{\lambda k_{2}{ }^{2}}\right) \sin \theta+\frac{\left(\frac{k_{2}}{k_{1}}\right)^{\prime} k_{1} \cos \theta^{\prime}}{\lambda k_{2} \cos \theta}-\lambda k_{2}{ }^{2} \gamma \gamma^{\prime}=0
\end{array}
$$

where ${\frac{k_{1}{ }^{*}}{\eta^{*}} s^{*}}=\left({\frac{k_{1}{ }^{*}}{k_{1}{ }^{2}+k_{2}{ }^{*}}}^{2}\right)^{\prime} \frac{1}{\cos \theta}$ and $\eta_{*} \frac{k_{1}{ }^{*}}{\eta^{*}}=\gamma \frac{\gamma^{\prime}}{\cos \theta}$. 
Theorem 5. Tangent vector fields of striction curves along normal ruled surface and Mannheim Darboux ruled surface are perpendicular under the condition

$$
\left(\left(\frac{\beta^{\prime}}{\cos \theta}\right)^{2}-\left(\frac{\beta^{\prime}}{\cos \theta}\right)^{\prime} \frac{\beta^{\prime}}{\cos ^{2} \theta} \frac{\beta \beta^{\prime}}{\cos \theta}\right)(a \cos \theta-c \sin \theta)+b\left(\frac{\beta^{\prime}}{\cos \theta}\right)^{\prime}=0
$$

Proof. Since $\left\langle T_{2},\left(T_{4}\right)^{*}\right\rangle=d^{*}(a \cos \theta-c \sin \theta)+b e^{*}$ and under the orthogonality condition $d^{*}(a \cos \theta-c \sin \theta)+b e^{*}=0$

$$
\begin{aligned}
\frac{\mu^{*}-\mu^{* \prime} \frac{k_{2}{ }^{*}}{k_{1}{ }^{*}}}{\mu^{*}\left\|c_{4}(s)\right\|^{*}}(a \cos \theta-c \sin \theta)+b \frac{\mu^{* \prime}}{\mu^{* 2}\left\|c_{4}{ }^{\prime}(s)\right\|^{*}}=0 & \Rightarrow \mu^{*}\left(\mu^{*}-\mu^{* \prime} \frac{k_{2}^{*}}{k_{1}{ }^{*}}\right)(a \cos \theta-c \sin \theta)+b \mu^{* \prime}=0 \\
& \Rightarrow\left(\left(\frac{\beta^{\prime}}{\cos \theta}\right)^{2}-\left(\frac{\beta^{\prime}}{\cos \theta}\right)^{\prime} \frac{\beta^{\prime}}{\cos ^{2} \theta} \frac{\beta \beta^{\prime}}{\cos \theta}\right)(a \cos \theta-c \sin \theta)+b\left(\frac{\beta^{\prime}}{\cos \theta}\right)^{\prime}=0 .
\end{aligned}
$$

where $\beta=\frac{k_{1}}{\lambda k_{2} \theta^{\prime}}$ and $\mu^{*}=\left(\frac{k_{2}{ }^{*}}{k_{1}{ }^{*}}\right)_{s^{*}}$.

Proposition 6. Tangent vector fields of striction curves along Darboux ruled surface and Mannheim normal ruled surface are perpendicular under the condition

$$
\tan \theta=-\frac{d\left(\frac{-\left(k_{1}{ }^{2}+k_{2}{ }^{2}\right)}{k_{2}}\right)^{2}+e \frac{k_{2}^{2} \theta^{\prime 2}+\cos ^{2} \theta\left(k_{1}{ }^{2}+k_{2}{ }^{2}\right)^{2}}{k_{2}{ }^{2} \cos ^{2} \theta}\left(\frac{\theta k_{2}{ }^{2} \cos \theta}{k_{2}{ }^{2} \theta^{\prime 2}+\cos ^{2} \theta\left(k_{1}{ }^{2}+k_{2}{ }^{2}\right)^{2}}\right)^{\prime} \frac{1}{\cos \theta}}{d \frac{k_{2}{ }^{2} \theta^{\prime 2}+\cos ^{2} \theta\left(k_{1}{ }^{2}+k_{2}{ }^{2}\right)^{2}}{k_{2}{ }^{2} \cos ^{2} \theta}\left(\frac{\theta k_{2}{ }^{2} \cos \theta}{k_{2}{ }^{2} \theta^{\prime 2}+\cos ^{2} \theta\left(k_{1}{ }^{2}+k_{2}{ }^{2}\right)^{2}}\right)^{\prime} \frac{1}{\cos \theta}+e\left(\frac{-\left(k_{1}{ }^{2}+k_{2}{ }^{2}\right)}{k_{2}}\right)^{2}} .
$$

Theorem 6. Tangent vector fields of striction curves along Darboux ruled surface andMannheim Darboux ruled surface are perpendicular under the condition; $\frac{\beta^{\prime}}{\cos \theta}-\left(\frac{\beta^{\prime}}{\cos \theta}\right)^{\prime}-\beta=0$ or $\tan \theta=\frac{d}{e}$.

Proof. Since $\left\langle T_{4},\left(T_{4}\right)^{*}\right\rangle=d^{*}(d \cos \theta-e \sin \theta)=0$.Then

$$
d \cos \theta-e \sin \theta=0 \Rightarrow \tan \theta=\frac{d}{e} \quad \text { or } \quad d^{*}=0 \Rightarrow \frac{\mu^{*}-\mu^{* \prime}-\frac{k_{2}{ }^{*}}{k_{1}{ }^{*}}}{\mu^{*}\left\|c_{4}{ }^{* \prime}(s)\right\|^{*}}=0, \quad \mu^{* \prime}{ }_{s^{*}}=\left(\frac{\beta^{\prime}}{\cos \theta}\right)^{\prime} \frac{1}{\cos \theta} .
$$

\section{Competing interests}

The authors declare that they have no competing interests.

\section{Authors' contributions}

All authors have contributed to all parts of the article. All authors read and approved the final manuscript.

\section{References}

[1] Do Carmo, M. P., Differential Geometry of Curves and Surfaces, Prentice-Hall, isbn 0-13-212589-7, 1976.

[2] Ergüt M., Körpınar T. and Turhan E., On Normal Ruled Surfaces of General Helices In The Sol Space Sol ${ }^{3}$, TWMS J. Pure Appl. Math., 4(2), 125-130, 2013.

[3] Graves L.K., Codimension one isometric immersions between Lorentz spaces, Trans. Amer. Math. Soc., 252, 367-392, 1979.

[4] Hacısalihoğlu H.H., Differential Geometry (in Turkish), 1994.

[5] Izumiya S., Takeuchi, N., Special curves and Ruled surfaces, Beitrage zur Algebra und Geometrie Contributions to Algebra and Geometry, 44(1), 203-212, 2003. 
[6] K1lıçoğlu Ş,On the Involute B-scrolls in the Euclidean Three-space $E^{3}$,Thirteenth International Conference on Geometry, 205-214, 2012.

[7] Kılıçoğlu Ş, Şenyurt S. and Hacısalihoğlu H.H., An examination on the positions of Frenet ruled surfaces along Bertrand pairs a and $a^{*}$ according to their normal vector fields in $E^{3}$, Applied Mathematical Sciences, 9(142), 7095-7103, 2015.

[8] Kilı̧̧oğlu Ş, Şenyurt S. and Ç alışkan A., On the striction curves along the involutive and Bertrandian Darboux ruled surfaces based on the tangent vector fields, NTMSCI 4, No. 4, 128-136, 2016.

[9] Kılıçoğlu Ş, Şenyurt S. and Hacısalihoğlu H.H., On the Striction Curves of Involute and Bertrandian Frenet Ruled Surfaces in E ${ }^{3}$, Applied Mathematical Sciences, 9(142), 7081-7094, 2015.

[10] Liu H. and Wang F., Mannheim partner curves in 3-space, Journal of Geometry, 88(1-2), 120-126(7), 2008.

[11] Orbay K. and Kasap E., On Mannheim partner curves in $E^{3}$,International Journal of Physical Sciences, 4(5), 261-264, 2009.

[12] Orbay K., Kasap E. and Aydemir Í, Mannheim Offsets of Ruled Surfaces, Hindawi Publishing CorporationMathematical Problems in Engineering, 9, 2009.

[13] Şenyurt S. and KKılıçoğlu Ş, On the differential geometric elements of the involute D scroll, Advances in Applied Clifford Algebras, 25(4), 977-988, 2015. 